\title{
EM08 mini-symposium on granular materials-editorial: interconnecting engineering and physics perspectives
}

\author{
K. M. Hill • Hayley Shen
}

Received: 25 January 2010 / Published online: 2 March 2010

(C) Springer-Verlag 2010

\section{Introduction}

The Engineering Mechanics Institute (EMI) was formed in 2007. Its predecessor was the Engineering Mechanics Division within the American Society of Civil Engineers. In view of the growing inter-disciplinary nature of engineering mechanics, the Institute was formed to serve the broader engineering community through the development and application of engineering mechanics. The mission of EMI is to anticipate and adapt to new challenges that will face tomorrow's engineers through creating an environment that encourages interactions of all science and technology fields. Each summer an institution-wide conference is held to promote the most innovative developments in the field of mechanics, regardless of the discipline of the ultimate user.

This Granular Materials Special Issue is based on a MiniSymposium held at the inaugural conference of EMI on May 18-21, 2008 at the University of Minnesota in Minneapolis, Minnesota.

K. M. Hill (更)

St. Anthony Falls Laboratory, Department of Civil Engineering, University of Minnesota, Minneapolis, MN 55414, USA

e-mail:kmhill@umn.edu

URL: www.ce.umn.edu/people/faculty/hill/

\section{H. Shen}

Department of Civil and Environmental Engineering,

Clarkson University, Potsdam, NY 13699-5710, USA

e-mail: hhshen@clarkson.edu

URL: http://people.clarkson.edu/ hhshen/
Meeting organizing committee:

- Dr. Steven Wojtkiewicz, Chair (UMN)

- Dr. Roberto Ballerini, co-Chair (UMN)

- Dr. Bojan Guzina, co-Chair (UMN)

Mini-symposium organizing committee:

- Dr. Kimberly Hill, co-Chair (UMN)

- Dr. Amy Rechenmacher, co-Chair (USC)

- Dr. Hayley Shen, co-Chair (Clarkson University)

\section{Theme of this special issue}

Fully characterizing granular materials remains a pressing challenge to scientists and engineers from many disciplines studying natural and industrial phenomena. For example, transitions between the fluid-like, solid-like, and gas-like behaviors are important for processing granular materials, but cannot be described using traditional models for solids, fluids and gases. While much progress has been made, there is still no suitable predictive model for much of the behavior of granular materials, including segregation, phase transitions, and the effects of interstitial fluids.

This mini-symposium brought together mathematicians, engineers, and physicists to discuss recent progress in certain key facets of granular materials research as well as current unsolved problems faced by scientists and engineers in the field. Much of granular materials research has benefited from the participation of researchers from multiple fields. To reflect this, the invited talks reflected areas that benefited from a multi-disciplinary approach. Topics of interest included the following four areas: (1) Transitions in granular materials (rapid to quasi-static regimes); (2) Particle-laden flow (particle/fluid flows); (3) Segregation; (4) 
Computational challenges for modeling transitions in granular materials.

\section{Invited papers}

Seven invited papers appear in this special issue that highlight research in these areas that was reported in the minisymposium. Two papers address the need for theoretical frameworks for dense sheared granular materials and granular-fluid systems. Two other papers describe physical experiments and associated analysis designed to determine certain physical details near the jamming point of relatively dense granular systems. The other three papers address certain complications related to interstitial fluids of granular-fluid systems and segregation.

A paper by Goddard highlights a general continuum framework for modeling granular systems based on the notion of parametric hypoplasticity. The theory allows for both Reynolds dilatency and strain-induced anisotropy. Jenkins and Berzi extended an existing form of kinetic theory of dense granular flows to one where the particles are more dissipative and frictional than was previously provided for by the theory. They do so in part by incorporating an additional length scale in the expression for the collisional rate of dissipation. The length scale is associated with the size of a cluster of correlated particles observed for denser systems where other forms of kinetic theory have been shown to break down.

Zhang et al. are concerned with microscopic properties of sheared granular materials near the jamming transition. Their experiments measure the forces throughout a sheared granular system of 2-d disks and from these microscopic measures determine macroscopic parameters such as the stress and fabric tensors and contact numbers. From probing the material in this way they provide insight to key components of sheared systems near the jamming transitions. Ramé et al. are concerned with the jamming transition for a much less idealized granular system. Their paper focuses on characterizing the flowability of the lunar soil simulant, JSC-1a with a particular focus on hopper design and use for lunar applications. They show the apparent best hopper design varies with the particular flowability test. Ultimately, both types of experiments will be necessary for the development of a complete granular theory and its practical applicability.
Ruiz-Angulo and Hunt address the need for certain collisional parameters for developing models in granular-fluid flows. In particular, the authors described experimental results for particles in viscous fluids where the impact of particles with flexible boundaries was measured. The results provide important information toward a more general model for granular fluid flows and have details of particular importance for the understanding of erosion due to slurries, a common problem in pipeline flow and in geophysical flows such as debris flows.

Anand et al. addresses the effect of a small amount of fluid on segregation of different sized granular materials. The particular problem is motivated by the desire to have powders well-mixed in the pharmaceutical industry. Anand et al. showed the relationship between cohesiveness and particle size could be put in terms of a non-dimensional number that could predict state of mixing. Hill et al. were concerned with another context where segregation was unwanted, here, in a new three-dimensional sensing system where tracer particles were designed to track local flow. Competing size and density segregation effects were investigated by Hill et al. for possible solution to a problem of a passive tracer particle that does not necessarily flow with the particles of interest.

These papers represent a sample of how the vibrancy of the granular community continues to grow as a result of research inspired by the problems from multiple disciplines and the rich variety of approaches this diverse community affords.

\section{The next EMI conference}

EMI will hold its next annual conference on August 8-11, 2010 at the University of Southern California in Los Angeles, CA. Dr. Roger Ghanem (Department of Aerospace and Mechanical Engineering) and Dr. Amy Rechenmacher (Department of Civil and Environmental Engineering) will be the Co-chairs of the EMI 2010 Conference.

Acknowledgments The authors would like to acknowledge helpful discussions with Profs. Stefan Luding and Bob Behringer in preparing this special issue. The authors would also like to acknowledge assistance from Prof. Amy Rechenmacher in organizing the special session. Hill would like to acknowledge funding from NSF grants CMMI-0625022, CBET-0756480, and CBET-0932735. 